\title{
Management of CNS metastases in small cell lung cancer: a consensus report
}

\author{
Anders G. Pedersen ${ }^{1}$, James F. Bishop ${ }^{2}$, Norman M. Bleehen ${ }^{3}$, Helena Earl ${ }^{4}$, \\ David S. Ettinger ${ }^{5}$, F. Anthony Greco ${ }^{6}$, Paul E.G. Kristjansen ${ }^{1}$, Pieter E. \\ Postmus $^{7}$ and Andrew T. Turrisi ${ }^{8}$ \\ ${ }^{1}$ Department of Oncology, Finsen Institute, Copenhagen (Denmark); ${ }^{2}$ Dept. of Cancer Medicine, Peter MacCallum \\ Cancer Institute, Melbourne (Australia); ${ }^{3}$ Department of Clinical Oncology and Radiotherapy, University of \\ Cambridge, Cambridge (UK.); ${ }^{4}$ Department of Radiotherapy and Oncology, University College Hospital, London \\ (U.K.); ${ }^{5}$ Oncology Center, Johns Hopkins University, Baltimore, MD (U.S.A.); ${ }^{6}$ Division of Oncology, Department of \\ Medicine, Vanderbilt University, Nashville, Tennessee (U.S.A); ${ }^{7}$ Department of Pulmonary Medicine, University \\ Hospital, Groningen (The Netherlands); ${ }^{8}$ and Department of Radiation Oncology, University of Michigan, Ann Arbor, \\ $M I$ (US.A.)
}

(Accepted 15 September 1989)

Introduction

Prevention and treatment of central nervous system (CNS) metastases remain a major problem in the treatment of small cell lung cancer (SCLC). CNS metastases are normally categorized into parenchymal (brain) metastases, leptomeningeal carcinomatosis and spinal cord compression. The incidence rises with the length of survival [1-3]. The methodological difficulties in establishing the true dimensions of the problem and in evaluating treatment results have been addressed recently elsewhere [4,5]. The present report summarizes information obtained since the last IASLC workshop consensus report [6]. It includes points of interest for future work along with guidelines, in so far as current knowledge permits recommendations to be made. In-depth analysis of the role of elective cranial irradiation (PCI), chemo- and radiotherapy of CNS metastases and sequelae of these treatments has been prepared in conjunction with the present workshop and can be found elsewhere in this issue [7-9].

\section{Elective cranial irradiation (PCI)}

Since the 1981 consensus report no additional trials on the value of $\mathrm{PCI}$ in patients obtaining a complete remission have been published. Retrospective evaluations of patients receiving and not receiving $\mathrm{PCI}$ have been published and reviewed elsewhere [5,7].

Correspondence: A.G. Pedersen, Dept. of Oncology, Rigshospitalet, Finsen Institute, 49 Strandboulevarden, DK-2100 Copenhagen, Denmark.

0169-5002/89/\$03.50 @ 1989 Elsevier Science Publishers B.V. (Biomedical Division) 
The available information suggests the following.

(1) Approximately $10 \%$ of patients who achieve a complete response and do not receive PCI develop brain metastases as the sole site of recurrent disease.

(2) PCI prolongs the period before occult brain metastases become symptomatic, but may not prevent their eventual occurrence.

(3) Patients receiving PCI may be at greater risk of developing late neuro-psychological complications.

Currently the value of $\mathrm{PCI}$ in patients who obtain a complete response is under investigation in two ongoing trials, but additional randomized trials are needed.

Apart from looking at whether or not PCI should be given, information on the proper timing and optimal dosage is important. In such studies, information on the type of chemotherapy administered, and whether it is given concurrently with the PCI should be included. In addition, the use of corticosteroids may be of importance. Patients should be evaluated before PCI and at preset intervals thereafter. The evaluation should include contrast enhanced brain CT-scans and some semi-quantitative determination of the cerebral function with an established methodology.

On the basis of current knowledge, the use of PCI in patients in complete remission is considered optional in the non-investigational setting. For patients who do not obtain a complete response there is little evidence to support the use of PCI.

\section{Treatment of CNS metastases}

Most questions concerning the treatment of CNS metastases raised in the 1981 consensus report unfortunately remain unanswered [6]. Additional points have emerged following a number of recent reports on the use of chemotherapy in the treatment of brain metastases [9]. The major problems in evaluating current treatment possibilities, such as e.g. definition of endpoints, selection of patients, and impact of concurrent treatment, have been addressed elsewhere $[5,9]$. These problems are partially a consequence of the retrospective nature of many of the studies.

A number of smaller investigations have reported chemotherapy of brain metastases to be efficacious, particularly in previously untreated patients [9]. In studies using conventional combination chemotherapy, brain metastases have responded at rates comparable to that found in other metastatic sites. Thus, the conception of the brain as a sanctuary is under reevaluation. Some of these trials have used chemotherapy alone in untreated patients. High-dose chemotherapy to patients relapsing with brain metastases has yielded some responses but at a cost of considerable toxicity [9]. In view of the expected short survival of such patients it is currently questionable if a possible therapeutic gain matches the adverse effects of such high-dose treatments.

Randomized trials evaluating the need for combined chemo- and radiotherapy in patients with newly diagnosed disease and brain metastases are needed. In addition, much information is needed on factors relevant to the evaluation of treatment outcome following radio- or chemotherapy. (1) What is the best measure of treatment effect? (2) What were the patients' characteristics of those receiving compared with those not receiving CNS treatment? (3) What is the diagnostic and therapeutic delay? (4) How many patients with brain metastases did not receive treatment? (5) How many of the patients were newly diagnosed patients with SCLC. (6) How many patients had disease progression outside the CNS? (7) At what time after the last chemotherapy were the brain metastases diagnosed? (8) Do corticosteroids influence the effect of chemotherapy through its influence on brain capillary permeability? Prospective studies on both chemo- and radiotherapy accounting for these aspects are needed. Follow-up should be made 
both with contrast enhanced CT-scan and evaluation of neurological function.

Treatment of leptomeningeal carcinomatosis is still disappointing. This may partially be due to the frequent occurrence of multiple metastases when leptomeningeal carcinomatosis is found. This factor along with the lack of reliable objective parameters to monitor treatment outcome makes the interpretation of various treatment approaches difficult. Intrathecal chemotherapy with methotrexate remains the most widely used treatment [9]. Only limited information is available on the intrathecal use of other cytostatic agents. Alternatively, high-dose systemic etoposide has been used with modest effect but again with considerable toxicity. Radiotherapy for bulky disease can be used, but irradiation to the whole neuraxis is only rarely indicated because of the risk of compromising the bone marrow capacity to withstand chemotherapy.

There were no new data on treatment of spinal cord compression available for discussion.

\section{Sequelae to CNS treatment}

Since the 1981 workshop a number of retrospective analyses on neuropsychologic complications in long-term survivors have appeared [8]. They lack pretherapeutic evaluation and often consist of patients who have received slightly different treatments. These reports have raised concern for possible adverse effects in patients surviving for longer periods following PCI. The contribution of different chemotherapeutic agents and the simultaneous administration of chemo- and radiotherapy is unclear. Aspects of these sequelae have been reviewed elsewhere in this issue [8]. Information on the quality of life in general and of the neuropsychological function in particular is needed in prospective studies. Furthermore an evaluation of PCI should also include contrast enhanced CT-scanning and nuclear magnetic resonance (NMR)-imaging to see if additional information on CNS toxicity can be obtained by these diagnostic methods.

\section{References}

1 Komaki, R., Cox, J.D. and Whitson, W. (1981) Risk of brain metastases from small cell carcinoma of the lung related to length of survival and prophylactic irradiation. Cancer Treat. Rep. 65: 811-814.

2 Nugent, J.L., Bunn, P.A., Matthews, M. et al. (1979) CNS metastases in Small Cell Bronchogenic carcinoma. Increasing frequency and changing pattern with lengthening of survival. Cancer 44: 1885-1893.

3 Pedersen, A.G. (1986) Diagnosis of CNS metastases from SCLC. In: Lung Cancer: Basic and Clinical Aspects (H.H. Hansen Ed.) Martinus Nijhoff Publishers, Boston, pp. 153-182.

4 Pedersen, A.G., Kristjansen, P.E.G. and Hansen, H.H. (1988) Prophylactic cranial irradiation and small cell lung cancer. Cancer Treat. Rev. 15: 85-103.

5 Kristjansen, P.E.G. and Pedersen, A.G. (1989) CNS therapy in small cell lung cancer. In: Basic and Clinical Concepts of Lung Cancer (H.H. Hansen Ed.), Kluwer, Boston, Pp. 275-299.

6 Bleehen, N.M., Bunn, P.A., Cox, J.D. et al. (1983) Role of radiation therapy in small cell anaplastic carcinoma of the lung. Cancer Treat. Rep. 67: 11-19.

7 Kristjansen, P.E.G. (1989) The role of cranial irradiation in the management of patients with small cell lung cancer. Lung Cancer, this issue.

8 Gregor, A. (1989) CNS toxicity. Lung Cancer, this issue.

9 Postmus, P.E., Sleijfer, D.T. and Haaxma-Reiche, H. (1989) Chemotherapy for central nervous system metastases from small cell lung cancer. A review. Lung Cancer, this issue. 